\title{
Características químicas, físicas e sensoriais de bolos de laranja e pães adicionados de inulina e oligofrutose
}

\section{Chemical, physical and sensory properties of orange cakes and breads with addition of inulin and oligofructose}

\author{
Patrícia Kelli de Souza-Borges ${ }^{1}$; Fabiana Ruriko Sokei ${ }^{1}$; Tâmara Denadai Spagnol ${ }^{1}$; \\ Ana Carolina Conti-Silva ${ }^{2 *}$
}

\begin{abstract}
Resumo
Inulina e oligofrutose são frutanos que, quando adicionados em alimentos, podem alterar importantes características dos produtos originais. Duas formulações de bolo de laranja (uma contendo 77,7g de inulina e outra $77,7 \mathrm{~g}$ de oligofrutose/inulina) e duas formulações de pão (uma contendo $130 \mathrm{~g}$ de inulina e outra $175 \mathrm{~g}$ de oligofrutose/inulina) foram comparadas com as respectivas formulações padrão (sem adição de frutanos) quanto à composição centesimal, textura, volume específico, cor e aceitação sensorial por meio da escala hedônica estruturada de nove pontos. Os bolos e pães com inulina e oligofrutose/ inulina apresentaram maior quantidade de fibra alimentar total em relação aos produtos padrão. $\mathrm{O}$ bolo de laranja com inulina apresentou menor coesividade e maior intensidade de amarelo no miolo em relação ao bolo padrão, sendo que a adição de inulina e oligofrutose/inulina reduziu a aceitação pela aparência, textura e sabor e a aceitação global em relação ao bolo padrão, embora a aceitação pelo aroma tenha sido igual para os três produtos. Os pães com inulina e oligofrutose/inulina foram mais duros e menos coesos do que o pão padrão, mas apenas o pão com inulina foi menos volumoso do que o padrão. $\mathrm{O}$ pão com inulina também apresentou casca com menor luminosidade, miolo com maiores intensidades de vermelho e de amarelo e maior saturação da cor em relação ao padrão, além de diferente tonalidade cromática. Em relação à aceitação sensorial, o pão com oligofrutose/inulina foi mais aceito quanto ao sabor e de forma global em relação ao pão padrão.
\end{abstract}

Palavras-chave: Frutanos, panificação, textura, cor, volume, aceitação sensorial

\begin{abstract}
Inulin and oligofructose are fructans that can change important characteristics in original products when added in food. Two formulations of orange cakes (one containing $77.7 \mathrm{~g}$ of inulin and another one $77.7 \mathrm{~g}$ of oligofructose/inulin) and two formulations of breads (one containing 130g of inulin and another one $175 \mathrm{~g}$ of oligofructose/inulin) were compared to the respective standard formulations (with no fructans) in relation to chemical composition, texture, volume, color and sensory acceptability with a nine-point hedonic scale. The cakes and breads with inulin and oligofructose/inulin showed greater amount of total dietary fiber in relation to standard products. The orange cake with inulin had lower cohesiveness and yellower dough when compared to the standard cake, but the addition of inulin and oligofructose/inulin reduced the acceptability for appearance, texture and flavor and the overall acceptability, although the acceptability for aroma was the same for the three products. The breads with inulin and oligofructose/ inulin were harder and less cohesive than the standard bread, and bread with inulin showed minor volume
\end{abstract}

\footnotetext{
${ }^{1}$ Discentes do Instituto de Biociências, Letras e Ciências Exatas, Universidade Estadual Paulista “Júlio de Mesquita Filho”, UNESP, São José do Rio Preto, SP. E-mail: patiksouza@globo.com; fabiana_sokei@hotmail.com; tamara.spagnol@yahoo.com.br

${ }^{2}$ Prof do Instituto de Biociências, Letras e Ciências Exatas, UNESP, São José do Rio Preto, SP. E-mail: contisil@ibilce.unesp.br

* Autor para correspondência
} 
in relation to the standard. The bread with inulin also presented crust with lower luminosity, dough with higher intensity of redness and yellowness and higher intensity of color in relation to standard, besides different hue. In relation to sensory acceptability, the bread with oligofructose/inulin had higher overall acceptability and flavor when compared to standard bread.

Key words: Fructans, bakery, texture, color, volume, sensory acceptability

\section{Introdução}

Prebióticos são compostos alimentares não digeridos pelas enzimas do trato gastrointestinal humano e que se comportam como fibras. Essas substâncias agem como substratos específicos para as bactérias benéficas, estimulando seletivamente a proliferação ou atividade de populações de bactérias desejáveis no cólon, como as bifidobactérias e os lactobacilos (probióticos) (MATTILASANDHOLM et. al., 2002; ROBERFROID, 2007). Pelo fato de os prebióticos apresentarem características funcionais semelhantes às fibras solúveis, eles são fermentados no intestino grosso por bactérias do cólon, produzindo ácido lático, ácidos graxos de cadeia curta (acético, propiônico e butírico) e gases, reduzindo assim o $\mathrm{pH}$ intestinal e inibindo a proliferação de microorganismos nocivos à saúde (WANG, 2009).

Inulina eoligofrutose são prebióticos pertencentes a uma classe de carboidratos chamada frutanos, termo genérico usado para descrever todos os oligo e polissacarídeos de frutose de origem vegetal. A inulina é um carboidrato de reserva encontrado principalmente na raiz da chicória (Cichorium intybus) e na alcachofra de Jerusalém (Helianthus tuberosus), e, estruturalmente, é composta por polímeros de $\beta$-D-frutofuranosil unidas por ligações do tipo $\beta(2 \rightarrow 1)$, com um grau de polimerização que pode chegar até 70 (MORRIS; MORRIS, 2012). Já a oligofrutose é obtida através da hidrólise parcial da inulina de chicória e subsequente purificação, sendo que seu grau de polimerização varia de 2 a 8 (BIEDRZYCKA; BIELECKA, 2004; ROBERFROID, 2005).

No Brasil, a Agência Nacional de Vigilância Sanitária aprova a alegação de que a inulina e a oligofrutose ajudam a equilibrar a flora intestinal, desde que seu consumo esteja associado a uma dieta equilibrada, a um estilo de vida saudável e que seja acompanhado da ingestão de líquidos, sendo necessário que porções de alimentos sólidos contenham, pelo menos, três gramas de inulina ou oligofrutose, ou ambos (ANVISA, 2012).

A inulina e a oligofrutose podem ser aplicadas em grande variedade de alimentos com as funções de fibra, prebióticos, substitutos de açúcar e gordura (FRANCK, 2002; WANG, 2009), sendo que os produtos de panificação representam um grupo de alimentos promissor para aplicação dessas substâncias, pois são consumidos mundialmente e apresentam longa estabilidade durante o armazenamento (LAMSAL; FAUBION, 2009). No entanto, a adição dessas substâncias em alimentos pode acarretar alterações nos produtos originais, principalmente mudanças físicas e sensoriais. Por isso, o objetivo deste trabalho foi avaliar parâmetros químicos e físicos e a aceitação sensorial de bolos de laranja e pães adicionados de inulina e oligofrutose.

\section{Material e Métodos}

\section{Material}

Os frutanos foram fornecidos pela BeneoOrafti (Tienen, Bélgica), sendo as informações sobre os produtos fornecidas pelo fabricante. O produto Orafti ${ }^{\circledR} \mathrm{GR}$ é composto por $\geq 90 \mathrm{~g} / 100 \mathrm{~g}$ de inulina (grau de polimerização médio $\geq 10$ ) e $\leq 10$ $\mathrm{g} / 100 \mathrm{~g}$ de glicose + frutose + sacarose. $\mathrm{O}$ produto Orafti ${ }^{\circledR}$ Synergy1 é composto por, aproximadamente, $46 \mathrm{~g}$ de oligofrutose, $46 \mathrm{~g}$ de inulina e $8 \mathrm{~g}$ de glicose + frutose + sacarose, o grau de polimerização variou entre 2 e 8 para oligofrutose e não foi informado para inulina. Os demais ingredientes foram adquiridos 
em mercado local e foram sempre da mesma marca.

Os bolos de laranja, tipo inglês, foram elaborados (Tabela 1) de forma que uma porção, definida em 60 g por Brasil (2003), contivesse a quantidade mínima de três gramas de frutanos totais exigida por Anvisa (2012) para alegação de prebióticos, sendo, portanto, adicionada uma sobredose de $20 \%$ de frutanos devido à perda durante processamentos térmicos (BÖHM et. al., 2005). Quatro testes preliminares foram realizados para ajustes nas formulações, pois os bolos apresentavam-se pouco expandidos e pegajosos. Por isso, foi necessário aumentar a quantidade de farinha de trigo no preparo dos bolos contendo frutanos, mas sem a adição de água. Para o preparo dos bolos, todos os ingredientes, com exceção do fermento químico, foram misturados em liquidificador, em velocidade intermediária, por um minuto. O fermento químico foi acrescentado e misturado à massa no modo pulsar do equipamento. A massa foi colocada em formas de alumínio e assada a $110{ }^{\circ} \mathrm{C}$ por quarenta minutos em forno Pasiani (modelo Turbo 240 Classic, Itajobi, Brasil) pré-aquecido por trinta minutos.

Tabela 1. Formulações dos bolos padrão (P), contendo inulina (I) e contendo oligofrutose/inulina (O/I).

\begin{tabular}{lccc}
\hline Ingredientes & Bolo P & Bolo I & Bolo O/I \\
\hline Farinha de trigo $(\mathrm{g})$ & 260 & 290 & 310 \\
Açúcar refinado $(\mathrm{g})$ & 398 & 398 & 398 \\
Suco de laranja comercial $(\mathrm{mL})$ & 250 & 250 & 250 \\
Óleo de soja (mL) & 200 & 200 & 200 \\
Ovo $(\mathrm{g})$ & 150 & 150 & 150 \\
Essência de laranja (gota) & 10 & 10 & 10 \\
Fermento químico (g) & 10 & 10 & 10 \\
Orafti ${ }^{\circledR}$ GR $(\mathrm{g})$ & - & $77,7^{*}$ & - \\
Orafti ${ }^{\circledR}$ Synergy1 $(\mathrm{g})$ & - & - & $77,7^{* *}$ \\
\hline
\end{tabular}

* Equivale a 26,8 \% em relação à farinha de trigo e 5,6 \% em relação à massa total do bolo.

** Equivale a 25,1 \% em relação à farinha de trigo e 5,5\% em relação à massa total do bolo.

Fonte: Elaboração dos autores.

Os pães, tipo caseiro, foram elaborados (Tabela 2) de forma que uma porção, definida em $50 \mathrm{~g}$ por Brasil (2003), contivesse a quantidade mínima de três gramas de frutanos totais (ANVISA, 2012). Para isso, também foram adicionadas sobredoses, sendo de 60 e $118 \%$ de frutanos nos pães com inulina e com oligofrutose/inulina, respectivamente. Observase maior sobredose para o pão com oligofrutose/ inulina em função da perda ocorrida durante a fermentação da oligofrutose. Foram realizados três testes preliminares para ajustes nas formulações, mas não foi necessária a alteração na quantidade dos ingredientes em comum para as três formulações. Para o preparo dos pães, todos os ingredientes, com exceção da farinha de trigo e dos frutanos, foram misturados em liquidificador na menor velocidade por dois minutos e adicionados à farinha de trigo e aos frutanos. Essa massa foi sovada em masseira Liemen (Caxias do Sul, Brasil) por dez minutos e deixada para descanso por quarenta minutos. Dividiu-se a massa em porções de $100 \mathrm{~g}$, que foram moldadas em modeladora Universo (modelo 0687, nível 2) e acondicionadas em formas individuais de alumínio previamente untadas com óleo e polvilhadas com farinha de trigo. Após descanso de noventa minutos, as massas foram assadas a $110^{\circ} \mathrm{C}$ por quinze minutos em forno Pasiani, pré-aquecido por trinta minutos. 
Tabela 2. Formulações dos pães padrão (P), contendo inulina (I) e contendo oligofrutose/inulina (O/I).

\begin{tabular}{lccc}
\hline Ingredientes & Pão P & Pão I & Pão O/I \\
\hline Farinha de trigo $(\mathrm{g})$ & 650 & 650 & 650 \\
Leite integral $(\mathrm{mL})$ & 230 & 230 & 230 \\
Óleo de soja $(\mathrm{mL})$ & 150 & 150 & 150 \\
Açúcar refinado $(\mathrm{g})$ & 75 & 75 & 75 \\
Fermento biológico $(\mathrm{g})$ & 30 & 30 & 30 \\
Sal refinado $(\mathrm{g})$ & 9 & 9 & 9 \\
Ovo $(\mathrm{g})$ & 200 & 200 & 200 \\
OraftißGR $(\mathrm{g})$ & - & $130^{*}$ & - \\
Orafti®Synergy1 $(\mathrm{g})$ & - & - & $175^{* *}$ \\
\hline
\end{tabular}

* Equivale a 20 \% em relação à farinha de trigo e $8,8 \%$ em relação à massa total do pão.

** Equivale a $27 \%$ em relação à farinha de trigo e $11,5 \%$ em relação à massa total do pão.

Fonte: Elaboração dos autores.

A sobredose de frutanos foi iniciada aos $20 \%$ em função da recomendação da Beneo-Orafti, mas a quantidade de frutanos totais nos produtos prontos sempre foi verificada por meio do método enzimático-colorimétrico proposto por McCleary e Rossiter (2004), utilizando o kit FRUCTAN HK (Megazyme, Ireland), para verificação da quantidade mínima de $3 \mathrm{~g}$ por porção.

\section{Composição centesimal}

Foi analisada a composição centesimal dos produtos: umidade por evaporação a $105^{\circ} \mathrm{C}$, proteína por micro-Kjeldhal (fator de conversão de 6,25), lipídeos através do método de extração por Soxhlet com éter de petróleo e cinzas através de incineração em mufla a $550{ }^{\circ} \mathrm{C}$ (AOAC, 1990); fibras solúveis e insolúveis pelo método enzimático-gravimétrico descrito por Prosky et al. (1988), utilizando o kit enzimático TDF100A-1KT (Sigma-Aldrich, USA); frutanos totais determinados por meio do método enzimático-colorimétrico proposto por McCleary e Rossiter (2004), utilizando o kit FRUCTAN HK (Megazyme, Ireland). Os frutanos, apesar de serem fibras solúveis, não foram detectados na análise de fibra pelo método de Prosky et al. (1988), provavelmente devido à perda durante a etapa de filtração para obtenção da fibra solúvel. Por isso, considerou-se fibra alimentar total como a somatória entre fibra insolúvel, fibra solúvel e frutanos totais. E, por fim, os carboidratos disponíveis foram calculados por diferença em relação à somatória de umidade, proteína, lipídeos, cinzas e fibra alimentar total.

Todas as análises foram realizadas em triplicata, com exceção de fibra alimentar que foi em duplicata.

\section{Análises físicas}

A Análise do Perfil de Textura (TPA) de dez amostras foi realizada em texturômetro TAXT2i (Stable Micro Systems, Godalming, Inglaterra). Os parâmetros utilizados foram: velocidade de pré-teste $1 \mathrm{~mm} / \mathrm{s}$; velocidade de teste $1 \mathrm{~mm} / \mathrm{s}$, velocidade de pós-teste $10 \mathrm{~mm} / \mathrm{s}$; compressão de $50 \%$ da altura da amostra; tempo entre as duas compressões de 5 s; probe cilíndrico de alumínio com diâmetro de 25 $\mathrm{mm}$ (P25). Os dados foram coletados pelo software Texture Expert. Foram analisados os parâmetros de dureza, coesividade, elasticidade e mastigabilidade (SZCZESNIAK, 2002).

Os produtos foram pesados em balança semianalítica e, em seguida, foram embrulhados em filme de PVC e colocados em uma caixa cheia de sementes de painço. O volume de painço deslocado para fora da caixa foi medido e se obteve o volume de cada produto. Por fim, o volume específico 
$\left(\mathrm{cm}^{3} / \mathrm{g}\right)$ dos produtos foi calculado pela razão entre o volume e o peso dos mesmos (PIZZINATTO; MAGNO; CAMPAGNOLLI, 1993).

A análise de cor foi realizada em triplicata em colorímetro Hunterlab, modelo Color Flex (Reston, Estados Unidos), utilizando iluminante D65 e observador $10^{\circ}$. Utilizou-se o sistema CIE-L*a*b* $\left(\mathrm{L}^{*}=\right.$ luminosidade; $\mathrm{a}^{*}=$ vermelho/verde; $\mathrm{b}^{*}=$ amarelo/azul), sendo também obtidos os valores de croma $\left(\mathrm{C}^{*}\right)$ e tonalidade cromática $\left(\mathrm{H}^{*}\right)$.

\section{Análise sensorial}

As análises sensoriais foram realizadas em Laboratório de Análise Sensorial, em cabines individuais sob luz branca e temperatura de 22 ${ }^{\circ} \mathrm{C}$. Os bolos e pães foram analisados em sessões diferentes. O projeto foi aprovado pelo Comitê de Ética em Pesquisa do Instituto de Biociências, Letras e Ciências Exatas da Universidade Estadual Paulista “Júlio de Mesquita Filho" (parecer de n 029/10). Foram empregados como provadores somente indivíduos que não apresentavam intolerância ou alergia aos ingredientes presentes nos produtos testados.

Em cada sessão de avaliação (bolo ou pão), cinquenta provadores não treinados receberam três amostras (padrão, com inulina, com oligofrutose/ inulina) codificadas com três dígitos, que foram apresentadas de forma monádica, balanceada e aleatorizada, e um copo com água à temperatura ambiente. A análise sensorial foi realizada em bloco completo e as amostras foram apresentadas em pratos brancos de plástico. Foi aplicado um teste afetivo de aceitação, com escala hedônica estruturada de nove pontos $(9=$ gostei extremamente; $5=$ não gostei nem desgostei e 1 = desgostei extremamente) para avaliar os atributos aparência, aroma, textura e sabor, além da aceitação global (MEILGAARD; CIVILLE; CARR, 2007).

\section{Análise estatística}

Para comparação de médias resultantes de amostras menores de 30 replicatas (composição centesimal e parâmetros físicos), foi utilizada estatística não paramétrica, sendo o teste de MannWhitney para frutanos totais (comparação entre duas médias) e Kruskal-Wallis seguido do teste de diferença de Dunn (comparação entre três médias). Já para comparação das médias sensoriais $(n=50)$, foi aplicada estatística paramétrica de análise de variância (ANOVA) seguida do teste de Tukey. Todas as análises foram realizadas no programa GraphPad Instat, versão 3.05 (GraphPad Software, Inc., San Diego, Estados Unidos), e foram consideradas diferenças significativas quando $\mathrm{p} \leq 0,05$.

\section{Resultados e Discussão}

Os bolos contendo inulina e oligofrutose/inulina não apresentaram diferença significativa entre si em relação a todos os componentes (Tabela 3). Apesar disso, o bolo adicionado de inulina apresentou menor teor lipídico e protéico em relação ao padrão, enquanto este apresentou-se mais úmido e com menor teor de cinzas do que o bolo com oligofrutose/inulina. Quanto ao teor de fibra, não houve diferença entre fibras insolúveis e solúveis entre os três bolos, mas a fibra alimentar total foi maior para os bolos com frutanos devido à adição da inulina e oligofrutose/inulina. 
Tabela 3. Composição centesimal dos produtos padrão (P), contendo inulina (I) e contendo oligofrutose/inulina (O/I) (dados médios $\pm \mathrm{dp}$ ).

\begin{tabular}{lcccccc}
\hline \multirow{2}{*}{ /100g } & \multicolumn{3}{c}{ Bolo de laranja } & \multicolumn{3}{c}{ Pão } \\
\cline { 2 - 7 } & $\mathbf{P}$ & $\mathbf{I}$ & $\mathbf{O} / \mathbf{I}$ & $\mathbf{P}$ & $\mathbf{I}$ & $\mathbf{O} / \mathbf{I}$ \\
\hline Umidade & $22,9 \pm 0,1^{\mathrm{a}}$ & $20,6 \pm 0,1^{\mathrm{ab}}$ & $20,5 \pm 0,1^{\mathrm{b}}$ & $17,7 \pm 0,4^{\mathrm{a}}$ & $18,1 \pm 0,3^{\mathrm{a}}$ & $18,5 \pm 0,7^{\mathrm{a}}$ \\
Cinzas & $0,6 \pm 0,0^{\mathrm{a}}$ & $0,7 \pm 0,0^{\mathrm{ab}}$ & $0,8 \pm 0,0^{\mathrm{b}}$ & $1,3 \pm 0,1^{\mathrm{a}}$ & $1,2 \pm 0,1^{\mathrm{a}}$ & $1,2 \pm 0,1^{\mathrm{a}}$ \\
Lipídeos & $20,8 \pm 0,1^{\mathrm{a}}$ & $17,9 \pm 0,1^{\mathrm{b}}$ & $18,4 \pm 0,1^{\mathrm{ab}}$ & $13 \pm 0,3^{\mathrm{a}}$ & $13,8 \pm 0,2^{\mathrm{a}}$ & $13,8 \pm 0,1^{\mathrm{a}}$ \\
Proteínas & $4,8 \pm 0,1^{\mathrm{a}}$ & $3,6 \pm 0,2^{\mathrm{b}}$ & $4 \pm 0,1^{\mathrm{ab}}$ & $12,2 \pm 0,0^{\mathrm{a}}$ & $10,3 \pm 0,0^{\mathrm{a}}$ & $10,1 \pm 0,1^{\mathrm{a}}$ \\
Fibra alimentar total* & $3,4^{*}$ & 11,4 & 11,8 & 11 & 14,4 & 14,8 \\
Fibra insolúvel & $2,7 \pm 0,3^{\mathrm{a}}$ & $1,7 \pm 0,4^{\mathrm{a}}$ & $2,6 \pm 0,3^{\mathrm{a}}$ & $9,1 \pm 0,1^{\mathrm{a}}$ & $3,5 \pm 0,8^{\mathrm{a}}$ & $3,2 \pm 0,2^{\mathrm{a}}$ \\
Fibra solúvel & $0,7 \pm 0,2^{\mathrm{a}}$ & $0,7 \pm 0,2^{\mathrm{a}}$ & $0,9 \pm 0,6^{\mathrm{a}}$ & $1,9 \pm 0,7^{\mathrm{a}}$ & $2,1 \pm 0,5^{\mathrm{a}}$ & $2,7 \pm 0,3^{\mathrm{a}}$ \\
Frutanos totais & - & $9,0 \pm 1,4^{\mathrm{A}}$ & $8,3 \pm 2,5^{\mathrm{a}}$ & - & $8,8 \pm 0,5^{\mathrm{a}}$ & $8,9 \pm 0,3^{\mathrm{A}}$ \\
Carboidratos disponíveis & 47,4 & 45,8 & 44,5 & 44,8 & 42,2 & 41,7 \\
\hline
\end{tabular}

* Somatória de fibra insolúvel, fibra solúvel e frutanos totais.

Letras minúsculas diferentes na mesma linha, para um mesmo tipo de produto, indicam médias estatisticamente diferentes pelo teste de Dunn $(\mathrm{p} \leq 0,05)$.

Letras maiúsculas iguais na mesma linha, para um mesmo tipo de produto, indicam médias estatisticamente iguais pelo teste de Mann-Whitney $(\mathrm{p}>0,05)$.

Fonte: Elaboração dos autores.

Em relação aos pães, não houve diferença na composição centesimal entre os três produtos, apesar de, novamente, a fibra alimentar total ter sido maior para os pães com frutanos (Tabela 3).

Os bolos de laranja adicionados de inulina e oligofrutose/inulina não apresentaram diferenças entre si em relação à textura e volume específico, com exceção da coesividade que foi menor para o bolo com inulina em relação ao bolo padrão (Tabela 4). Da mesma forma, os dois pães adicionados de frutanos foram menos coesos do que o pão padrão. A menor coesividade indica menor força necessária para esticar um alimento até que este seja rompido (SZCZESNIAK, 2002), sendo assim, o bolo com inulina e os pães com frutanos apresentaram menor força para serem rompidos do que o bolo padrão, podendo ser considerados mais farinhentos. Frutos et al. (2008) também observaram queda na coesividade de pães adicionados de $12 \%$ de fibra de alcachofra.

Ainda, os dois pães adicionados de frutanos foram mais duros do que o pão padrão, enquanto apenas o pão com inulina foi menos volumoso do que este (Tabela 4). A adição de frutanos em produtos de panificação pode causar a diluição das proteínas formadoras de glúten, afetando a absorção de água pela massa e dificultando o desenvolvimento do glúten, conferindo maior dureza ao produto (COLLAR; SANTOS; ROSELL, 2007; ANGIOLONI; COLLAR, 2009). Resultados semelhantes foram encontrados por O'Brien et al. (2003), em que altas concentrações de inulina resultaram em maior dureza da casca de pães em relação aos pães contendo gordura, e por Ronda et al. (2005), em que a oligofrutose aumentou a firmeza de bolos em relação aos com sacarose. A redução de volume no pão contendo inulina corrobora os resultados encontrados por Wang, Rosell e Barber (2002) e Gomez et al. (2003). Segundo estes autores, a interação entre fibras e glúten impede a expansão da massa, devido ao escape do gás durante a fermentação e o assamento, reduzindo o volume do pão. Além disso, revisão feita por Morris e Morris (2012) aponta o aumento na dureza, medida por TPA, e decréscimo no volume de pães adicionados de oligofrutose e inulina em vários trabalhos. 
Tabela 4. Parâmetros de textura, cor e volume específico dos produtos padrão (P), contendo inulina (I) e contendo oligofrutose/inulina (O/I) (dados médios \pm dp).

\begin{tabular}{|c|c|c|c|c|c|c|}
\hline & \multicolumn{3}{|c|}{ Bolo de laranja } & \multicolumn{3}{|c|}{ Pão } \\
\hline & $\mathbf{P}$ & I & $\mathbf{O} / \mathbf{I}$ & $\mathbf{P}$ & I & $\mathbf{O} / \mathbf{I}$ \\
\hline \multicolumn{7}{|l|}{ Textura } \\
\hline Dureza $(N)$ & $0,9 \pm 0,3^{\mathrm{a}}$ & $1,1 \pm 0,5^{\mathrm{a}}$ & $1,1 \pm 0,2^{\mathrm{a}}$ & $2,0 \pm 0,2^{\mathrm{a}}$ & $3,9 \pm 1,4^{b}$ & $3,3 \pm 0,6^{\mathrm{b}}$ \\
\hline Coesividade & $0,7 \pm 0,1^{\mathrm{b}}$ & $0,3 \pm 0,2^{\mathrm{a}}$ & $0,5 \pm 0,2^{\mathrm{ab}}$ & $0,6 \pm 0,03^{b}$ & $0,3 \pm 0,04^{\mathrm{a}}$ & $0,4 \pm 0,1^{\mathrm{a}}$ \\
\hline Elasticidade & $0,8 \pm 0,1^{\mathrm{a}}$ & $0,8 \pm 0,1^{\mathrm{a}}$ & $0,8 \pm 0,1^{\mathrm{a}}$ & $0,8 \pm 0,1^{\mathrm{a}}$ & $0,7 \pm 0,1^{\mathrm{a}}$ & $0,7 \pm 0,1^{\mathrm{a}}$ \\
\hline Mastigabilidade (N) & $0,5 \pm 0,2^{\mathrm{a}}$ & $0,3 \pm 0,3^{\mathrm{a}}$ & $0,5 \pm 0,2^{\mathrm{a}}$ & $0,9 \pm 0,1^{\mathrm{a}}$ & $1,0 \pm 0,3^{\mathrm{a}}$ & $0,9 \pm 0,1^{\mathrm{a}}$ \\
\hline Volume específico $\left(\mathrm{cm}^{3} / \mathrm{g}\right)$ & $2,0 \pm 0,2^{\mathrm{a}}$ & $2,0 \pm 0,1^{\mathrm{a}}$ & $2,3 \pm 0,3^{\mathrm{a}}$ & $4,7 \pm 0,2^{\mathrm{b}}$ & $3,7 \pm 0,2^{\mathrm{a}}$ & $4,4 \pm 0,0^{\mathrm{ab}}$ \\
\hline \multicolumn{7}{|l|}{ Cor da casca } \\
\hline$L^{*}$ & $51,4 \pm 1,9^{\mathrm{a}}$ & $41,8 \pm 1,8^{\mathrm{a}}$ & $50,5 \pm 5,6^{\mathrm{a}}$ & $58,1 \pm 0,4^{\mathrm{b}}$ & $53,1 \pm 1,4^{\mathrm{a}}$ & $55,7 \pm 1,0^{\mathrm{ab}}$ \\
\hline$a^{*}$ & $18,6 \pm 0,7^{\mathrm{a}}$ & $20,5 \pm 0,6^{\mathrm{a}}$ & $18,7 \pm 1,7^{\mathrm{a}}$ & $12,9 \pm 0,3^{\mathrm{a}}$ & $15,5 \pm 1,4^{\mathrm{a}}$ & $14,5 \pm 1,0^{\mathrm{a}}$ \\
\hline$b^{*}$ & $38,9 \pm 5,3^{\mathrm{a}}$ & $30,6 \pm 1,5^{\mathrm{a}}$ & $36,6 \pm 3,7^{\mathrm{a}}$ & $36,7 \pm 0,6^{\mathrm{a}}$ & $35,1 \pm 0,8^{\mathrm{a}}$ & $34,6 \pm 0,6^{\mathrm{a}}$ \\
\hline$C^{*}$ & $43,2 \pm 4,7^{\mathrm{a}}$ & $36,8 \pm 1,1^{\mathrm{a}}$ & $41,2 \pm 2,6^{\mathrm{a}}$ & $38,9 \pm 0,4^{\mathrm{a}}$ & $38,4 \pm 1,2^{\mathrm{a}}$ & $37,5 \pm 0,9^{\mathrm{a}}$ \\
\hline$h$ & $64,2 \pm 3,5^{\mathrm{a}}$ & $56,2 \pm 1,7^{\mathrm{a}}$ & $62,8 \pm 4,3^{\mathrm{a}}$ & $70,7 \pm 0,6^{\mathrm{a}}$ & $66,2 \pm 1,6^{\mathrm{a}}$ & $67,3 \pm 1,2^{\mathrm{a}}$ \\
\hline \multicolumn{7}{|l|}{ Cor do miolo } \\
\hline$L^{*}$ & $77,6 \pm 0,3^{\mathrm{a}}$ & $74,3 \pm 0,7^{\mathrm{a}}$ & $74,6 \pm 0.5^{\mathrm{a}}$ & $68,8 \pm 0,8^{\mathrm{a}}$ & $69,5 \pm 1,0^{\mathrm{a}}$ & $76,0 \pm 1,0^{\mathrm{a}}$ \\
\hline$a^{*}$ & $2,8 \pm 0,3^{\mathrm{a}}$ & $4,7 \pm 0,4^{\mathrm{a}}$ & $4,4 \pm 0,1^{\mathrm{a}}$ & $0,1 \pm 0,0^{\mathrm{a}}$ & $1,2 \pm 0,3^{\mathrm{b}}$ & $0,8 \pm 0,0^{\mathrm{ab}}$ \\
\hline$b^{*}$ & $27,9 \pm 0,7^{\mathrm{a}}$ & $33,5 \pm 0,3^{b}$ & $30,5 \pm 0,7^{\mathrm{ab}}$ & $18,0 \pm 0,7^{\mathrm{a}}$ & $22,0 \pm 0,8^{b}$ & $20,4 \pm 0,4^{\mathrm{ab}}$ \\
\hline$C^{*}$ & $28,1 \pm 0,7^{\mathrm{a}}$ & $33,8 \pm 0,3^{\mathrm{a}}$ & $30,8 \pm 0,7^{\mathrm{a}}$ & $18,0 \pm 0,7^{\mathrm{a}}$ & $22,0 \pm 0,8^{b}$ & $20,4 \pm 0,4^{\mathrm{ab}}$ \\
\hline$h$ & $84,3 \pm 0,4^{\mathrm{a}}$ & $82,0 \pm 0,7^{\mathrm{a}}$ & $81,8 \pm 0,2^{\mathrm{a}}$ & $89,8 \pm 0,1^{\mathrm{b}}$ & $86,8 \pm 0,6^{\mathrm{a}}$ & $87,7 \pm 0,8^{\mathrm{ab}}$ \\
\hline
\end{tabular}

Letras diferentes na mesma linha, para um mesmo tipo de produto, indicam médias estatisticamente diferentes pelo teste de Dunn $(\mathrm{p} \leq 0,05)$.

Fonte: Elaboração dos autores.

Em relação à cor dos produtos, o bolo contendo inulina apresentou maior intensidade de amarelo (b*) do miolo em relação ao bolo padrão, enquanto que o pão contendo inulina apresentou casca com menor luminosidade $\left(\mathrm{L}^{*}\right)$ e miolo com maiores intensidades de vermelho $\left(\mathrm{a}^{*}\right)$ e de amarelo $\left(\mathrm{b}^{*}\right)$ e maior saturação $\left(C^{*}\right)$ em relação ao pão padrão, além de diferente tonalidade cromática (Tabela 4). Já o bolo e pão com oligofrutose/inulina não se diferenciaram nem dos produtos padrão nem dos produtos com inulina em relação à cor. Os frutanos, por serem constituídos de polímeros de frutose e a frutose ser um açúcar redutor, podem ter favorecido a reação de Maillard, contribuindo para o escurecimento do miolo e da casca dos produtos (AMREIN et al., 2004; DAMODARAN; PARKIN; FENNEMA, 2008). No entanto, os resultados são opostos aos encontrados por Peressini e Sensidoni (2009), que observaram que inulinas de cadeia curta resultaram em cores mais escuras, uma vez que possuem mais frutanos de baixo peso molecular. No atual trabalho, o escurecimento significativo, em relação aos produtos padrão, foi com a adição de inulina apenas, e não de oligofrutose/inulina.

A adição de inulina e oligofrutose/inulina aos bolos reduziu a aceitação pela aparência, textura e sabor e a aceitação global em relação ao bolo padrão, embora a aceitação pelo aroma tenha sido igual para os três produtos (Tabela 5). Apesar disso, a porcentagem mínima de notas acima de 6, que indica "gostei ligeiramente", foi de $92 \%$ para o aroma do bolo padrão, $82 \%$ para aroma e textura do bolo com inulina e $82 \%$ para o sabor do bolo com oligofrutose/inulina (Figura 1). A aceitação sensorial pelos bolos neste trabalho foi superior ou igual a outros trabalhos que estudaram a aceitação por bolos adicionados de frutanos (DEVEREUX et. al., 2003; RONDA et al., 2005; RODRÍGUEZGARCÍA et al., 2012). 
Figura 1. Porcentagem de notas acima de 6 ("gostei ligeiramente") atribuídas aos produtos padrão (P), contendo inulina (I) e contendo oligofrutose/inulina (O/I).
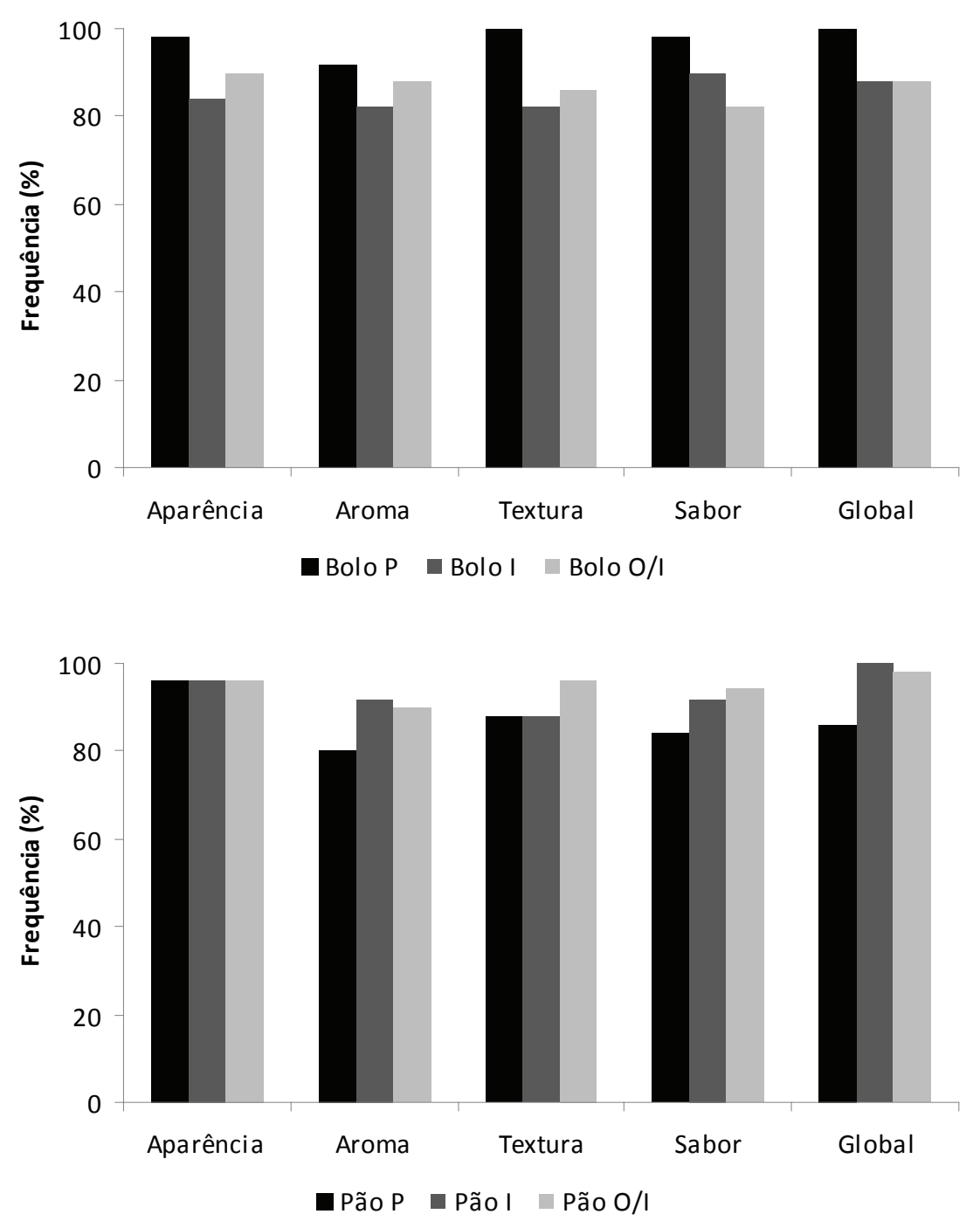

Fonte: Elaboração dos autores.

Tabela 5. Aceitação sensorial dos produtos padrão (P), contendo inulina (I) e contendo oligofrutose/inulina $(\mathrm{O} / \mathrm{I})$ (dados médios $\pm \mathrm{dp}$ ).

\begin{tabular}{ccccccc}
\hline & \multicolumn{2}{l}{ Bolo de laranja } & \multicolumn{3}{c}{ Pão } \\
\cline { 2 - 7 } & $\mathbf{P}$ & $\mathbf{I}$ & $\mathbf{O} / \mathbf{I}$ & $\mathbf{P}$ & $\mathbf{I}$ & $\mathbf{O} / \mathbf{I}$ \\
\hline Aparência & $7,9 \pm 1,0^{\mathrm{a}}$ & $7,1 \pm 1,5^{\mathrm{b}}$ & $7,2 \pm 1,4^{\mathrm{b}}$ & $7,7 \pm 1,1^{\mathrm{a}}$ & $7,8 \pm 1,0^{\mathrm{a}}$ & $8,0 \pm 1,0^{\mathrm{a}}$ \\
Aroma & $7,3 \pm 1,2^{\mathrm{a}}$ & $7,1 \pm 1,5^{\mathrm{a}}$ & $7,2 \pm 1,4^{\mathrm{a}}$ & $6,9 \pm 1,6^{\mathrm{a}}$ & $7,4 \pm 1,4^{\mathrm{a}}$ & $7,4 \pm 1,6^{\mathrm{a}}$ \\
Textura & $8,1 \pm 0,8^{\mathrm{a}}$ & $6,9 \pm 1,5^{\mathrm{b}}$ & $7,0 \pm 1,5^{\mathrm{b}}$ & $7,3 \pm 1,3^{\mathrm{a}}$ & $7,2 \pm 1,5^{\mathrm{a}}$ & $7,7 \pm 1,2^{\mathrm{a}}$ \\
Sabor & $7,7 \pm 1,1^{\mathrm{a}}$ & $6,9 \pm 1,4^{\mathrm{b}}$ & $6,9 \pm 1,7^{\mathrm{b}}$ & $6,8 \pm 1,8^{\mathrm{a}}$ & $7,4 \pm 1,3^{\mathrm{ab}}$ & $7,6 \pm 1,2^{\mathrm{b}}$ \\
Aceitação global & $8,0 \pm 0,8^{\mathrm{a}}$ & $7,1 \pm 1,3^{\mathrm{b}}$ & $7,0 \pm 1,6^{\mathrm{b}}$ & $7,0 \pm 1,6^{\mathrm{a}}$ & $7,6 \pm 1,0^{\mathrm{ab}}$ & $7,8 \pm 1,0^{\mathrm{b}}$ \\
\hline
\end{tabular}

Letras minúsculas diferentes na mesma linha, para um mesmo tipo de produto, indicam médias estatisticamente diferentes pelo teste de Tukey $(\mathrm{p} \leq 0,05)$.

Fonte: Elaboração dos autores. 
Já para os pães, a adição de oligofrutose/inulina aumentou a aceitação pelo sabor e aceitação global do pão em relação ao pão padrão, sendo o pão com inulina igualmente aceito ao pão padrão em relação a todos os atributos e também de forma global (Tabela 5). Além disso, a porcentagem mínima de notas acima de 6, que indica "gostei ligeiramente", foi de $80 \%$ para o aroma do pão padrão, 88 \% para a textura do pão com inulina e $90 \%$ para o aroma do pão com oligofrutose/inulina (Figura 1). A aceitação pelos pães testados neste estudo foi superior aos resultados de aceitação por pães adicionados de inulina relatados na literatura (WANG; ROSELL; BARBER, 2002).

\section{Conclusão}

A adição de inulina e oligofrutose/inulina a bolos de laranja e pães aumenta a quantidade de fibra alimentar total nos produtos. Para os bolos de laranja, a adição de inulina reduz a coesividade e aumenta a intensidade da tonalidade amarela do miolo em relação ao bolo padrão, e a adição de inulina e oligofrutose/inulina reduz a aceitação pela aparência, textura e sabor e a aceitação global em relação ao bolo padrão, embora a aceitação pelo aroma tenha sido igual para os três produtos. Em relação aos pães, a inulina causa alterações na cor e redução no volume específico, enquanto que ambos os frutanos resultam em pães mais duros e menos coesos do que o pão padrão. No entanto, a aceitação global e do sabor é maior para o pão adicionado de oligofrutose/inulina em relação ao padrão, mas igual ao pão contendo inulina.

\section{Agradecimentos}

Os autores agradecem à FAPESP (Fundação de Amparo à Pesquisa do Estado de São Paulo) (processos 2010/00996-0 e 2010/12211-8) e à PróReitoria de Pesquisa da Unesp pelo financiamento e à BENEO-Orafti pelo fornecimento dos frutanos.

\section{Referências}

AMREIN, T. M.; SCHÖNBÄCHLER, B.; ESCHER, F.; AMADO, R. Acrylamide in gingerbread: critical factors for formation and possible ways for reduction. Journal of Agricultural and Food Chemistry, Washington, v. 52, n. 13, p. 4282-4288, 2004.

ANGIOLONI, A.; COLLAR, C. Gel, dough and fibre enriched fresh breads: Relationships between quality features and staling kinetics. Journal of Food Engineering, Essex, v. 91, n. 4, p. 526-532, 2009.

ANVISA. Agência Nacional de Vigilância Sanitária. Alimentos/alimentos com alegações de propriedades funcionais e ou de saúdelalegações de propriedade funcional aprovadas. 2012. Disponível em $<\mathrm{http}$ ://www. anvisa.gov.br>. Acesso em: 04 dez. 2012.

ASSOCIATION OF OFFICIAL ANALYTICAL CHEMISTS - AOAC. Official methods of analysis. Washington: AOAC International, 1990.

BIEDRZYCKA, E.; BIELECKA, M. Prebiotic effectiveness of fructans of different degrees of polymerization. Trends in Food Science and Technology, Cambridge, v. 15, n. 3, p. 170-175, 2004.

BÖHM, A.; KAISER, I.; TREBSTEIN, A.; HENLE, T. Heat-induced degradation of inulin. European Food Research and Technology, Berlin, v. 220, n. 5-6, p. 466471, 2005.

BRASIL. Agência Nacional de Vigilância Sanitária. Resolução RDC no 359, de 23 de dezembro de 2003. Regulamento técnico de porções de alimentos embalados para fins de rotulagem nutricional. Diário Oficial [da] República Federativa do Brasil, Brasília, DF, 26 dez. 2003. Seção 1, p. 28-32.

COLLAR, C.; SANTOS, E.; ROSELL, M. Assessment of rheological profile of fibre enriched bread doughs by response surface methodology. Journal of Food Engineering, Essex, v. 78, n. 3, p. 820-826, 2007.

DAMODARAN, S.; PARKIN, K. L.; FENNEMA, O. R. Fennema's food chemistry. $4^{\text {th }}$ ed. Boca Raton: CRC Press, 2008.

DEVEREUX, H. M.; JONES, G. P.; MCCORMACK, L.; HUNTER, W. C. Consumer acceptability of low fat foods containing inulin and oligofructose. Journal of Food Science, Chicago, v. 68, n. 5, p. 1850-1854, 2003.

FRANCK, A. Technological functionality of inulin and oligofructose. British Journal of Nutrition, London, v. 87, p. S287-S291, 2002. Supplement 2.

FRUTOS, M. J.; GUILABERT-ANTON, L.; TOMASBELLIDO, A.; HERNANDEZ-HERRERO, J. A. Effect 
of artichoke (Cynara scolymus L.) fiber on textural and sensory qualities of wheat bread. Food Science and Technology International, London, v. 14, n. 5, p. 49-55, 2008.

GOMEZ, M.; RONDA, F.; BLANCO, C.; CABALLERO, P.; APESTEGUIA, A. Effect of dietary fibre on dough rheology and bread quality. European Food Research and Technology, v. 216, n. 1, p. 51-56, 2003.

LAMSAL, B. P.; FAUBION, J. M. The beneficial use of cereal and cereal components in probiotic foods. Food Reviews International, New York, v. 25, n. 2, p. 103-114, 2009.

MATTILA-SANDHOLM, T.; MYLLÄRINEN, P.; CRITTENDEN, R.; MOGENSEN, G.; FONDÉN, R.; SAARELA, M. Technological challenges for future probiotic foods. International Dairy Journal, Edmonton, v. 12, n. 2, p. 173-182, 2002.

MCCLEARY, B. V.; ROSSITER, P. Measurement of novel dietary fibers. Journal of the Association of Official Analytical Chemists, Washington, v. 87, n. 3, p. 707-717, 2004.

MEILGAARD, M.; CIVILLE, G. V.; CARR, B. T. Sensory evaluation techniques. $4^{\text {th }}$ ed. Boca Raton: CRC Press. 2007.

MORRIS, C.; MORRIS, G. A. The effect of inulin and fructo-oligosaccharide supplementation on the textural, rheological and sensory properties of bread and their role in weight management: a review. Food Chemistry, London, v. 133, n. 2, 237-248, 2012.

O'BRIEN, C. M. O.; MUELLER, A.; SCANNELL, A. G. M.; ARENDT, E. K. Evaluation of the effects of fat replacers on the quality of wheat bread. Journal of Food Engineering, Essex, v. 56, n. 2-3, p. 265-267, 2003.

PERESSINI, D.; SENSIDONI, A. Effect of soluble dietary fibre addition on rheological and breadmaking properties of wheat doughs. Journal of Cereal Science, London, v. 49, n. 2, p. 190-201, 2009.
PIZZINATTO, A.; MAGNO, C. P. R.; CAMPAGNOLLI, D. M. F. Avaliação tecnológica de produtos derivados da farinha de trigo pão, macarrão, biscoitos. Campinas: ITAL, 1993.

PROSKY, L.; ASP, N. G.; SCHWEIZER, T. F.; DEVRIES, J. W.; FURDA, I. Determination of insoluble, soluble and total dietary fiber in foods and foods products: intralaboratory study. Journal of the Association of Official Analytical Chemists, Washington, v. 71, n. 5, p. 1017-1023, 1988.

ROBERFROID, M. B. Prebiotics: the concept revisited. The Journal of Nutrition, Bethesda, v. 137, n. 11, p. 830837, 2007.

Introducing inulin-type fructans. British Journal of Nutrition, London, v. 93, p. S13-S25, 2005. Supplement 1.

RODRÍGUEZ-GARCÍA, J.; PUIG, A.; SALVADOR, A.; HERNANDO, I. Optimization of a sponge cake formulation with inulin as fat replacer: structure, physicochemical, and sensory properties. Journal of Food Science, Chicago, v. 77, n. 2, p. C189-C197, 2012.

RONDA, F.; GÓMEZ, M.; BLANCO, C. A.; CABALLERO, P. A. Effects of polyols and nondigestible oligosaccharides on the quality of sugar-free sponge cakes. Food Chemistry, Washington, v. 90, n. 4, p. 549555, 2005.

SZCZESNIAK, A. S. Texture is a sensory property. Food Quality and Preference, Barking, v. 13, n. 4, p. 215-225, 2002.

WANG, J.; ROSELL, C. M.; BARBER, C. B. Effect of the addition of different fibres on wheat dough performance and bread quality. Food Chemistry, Washington, v. 79, n. 2, p. 221-226, 2002.

WANG, Y. Prebiotics: present and future in food science and technology. Food Research International, Barking, v. 42, n. 1, p. 8-12, 2009. 